\title{
Neurobrucellosis Related to the Consumption of Unpasteurized Camel Milk in Ras Al Khaimah, United Arab Emirates
}

\author{
Omar Farooq AINahhas ${ }^{1}$ D , Godfred Antony Menezes ${ }^{2 *}(\mathbb{D}$, Khaled Alaboud \\ Alkheder $^{1}$ (D), Yousif Al Khafaji ${ }^{1}$ (D) and Nadeem Javid ${ }^{3}$ (D)
}

${ }^{1}$ RAK College of Medical Sciences (RAKCOMS), RAK Medical \& Health Sciences University (RAKMHSU), Ras Al Khaimah, UAE. ${ }^{2}$ Associate Professor \& Clinical Microbiologist, RAK College of Medical Sciences (RAKCOMS), RAK Medical \& Health Sciences University (RAKMHSU), Ras Al Khaimah, UAE. ${ }^{3}$ Ibrahim Bin Hamad Obaidullah (IBHO) Hospital, Ras Al Khaimah, UAE.

\begin{abstract}
Brucellosis is a zoonotic infection with more than 500,000 infections per year worldwide. Brucella spp. are transmitted from animals to humans by ingestion of infected food products, direct contact with an infected animal, or inhalation of aerosols. Brucellosis is considerably rare in United Arab Emirates (UAE). The objective of this manuscript is to report a case of neurobrucellosis in Ras Al-Khaimah. UAE. A 33-year-old male patient presented with fever, chills, rigors, myalgia, arthralgia, and neck stiffness after ingestion of unpasteurized raw camel milk. The patient was admitted to the hospital with brucellosis with possible CNS involvement. During hospitalization, patient developed testicular pain, diagnosed as orchitis. The patients clinical condition progressed from human brucellosis to neurobrucellosis confirmed by investigations.
\end{abstract}

Keywords: Brucellosis, zoonotic disease, contaminated food, Brucella spp

*Correspondence: godfred@rakmhsu.ac.ae; Tel.: +971 543266244.

(Received: January 27, 2020; accepted: March 25, 2020)

Citation: Omar Farooq AINahhas, Godfred Antony Menezes, Khaled alaboud alkheder, Yousif Al Khafaji, Nadeem Javid, Neurobrucellosis Related to the Consumption of Unpasteurized Camel Milk in Ras Al Khaimah, United Arab Emirates, J. Pure Appl. Microbiol., 2020; 14(1):183-187. https://doi.org/10.22207/JPAM.14.1.19

(C) The Author(s) 2020. Open Access. This article is distributed under the terms of the Creative Commons Attribution 4.0 International License which permits unrestricted use, sharing, distribution, and reproduction in any medium, provided you give appropriate credit to the original author(s) and the source, provide a link to the Creative Commons license, and indicate if changes were made. 


\section{INTRODUCTION}

Brucellosis is a zoonotic infection caused by the bacterial genus Brucella spp. The organism causes beyond 500,000 infections per year worldwide. The bacteria are transmitted from animals to humans by consumption of infected food products, straight contact with an infected animal, or inhalation of aerosols ${ }^{1}$. Human brucellosis is an important occupational hazard particularly in rural areas. We need to raise awareness about zoonotic brucellosis through educational programs and carry out regular screening of the animals for infectious diseases ${ }^{2,3}$. Brucella spp. has been detected in commercialized traditional cheese as well. There should be guidelines for good practices to be followed in such cheese manufacturing ${ }^{4}$.

Breaks in the epithelium, mucous membranes, conjunctivae, respiratory and gastrointestinal (GI) tracts can all serve as portals of entry to the bacteria. Once the organism gains entry to the bloodstream, the bacteria rapidly become intracellular pathogens confined inside circulating polymorphonuclear cells (PMNs) and macrophages ${ }^{5,6}$ Brucellae may replicate locally or they affect the nervous system either by direct methods or indirect methods, through cytokine or endotoxin release on the neural tissue, the infection initiates an immune response that leads to demyelination of neurons in the Central Nervous System (CNS) and Peripheral Nervous System (PNS) ${ }^{7}$.

Systemic brucellosis is a complication of neurobrucellosis infection. The occurrence of neurobrucellosis has been reported to be $5-7 \%$ in the literature ${ }^{5}$. Neurological complications include meningoencephalitis, encephalitis, myelitis, radiculitis, peripheral and cranial neuropathies, subarachnoid hemorrhage, brain abscess, psychiatric manifestations, and demyelinating syndrome $e^{5,6}$. The highest prevalence of neurobrucellosis is in the Mediterranean basin and the Arabian Peninsula. In the UAE the overall report rate was 3.3 per 100,000 population but greater rates were seen in certain population subgroups particularly expatriate males of working age in the Eastern Region (about 10 per 100,000) and UAE nationals of both genders and all ages in Abu Dhabi (between 4 - 24 per 100,000) ${ }^{1}$. The objective of this manuscript is to discuss a case of 33-year-old male presented with neurobrucellosis encountered in in Ras Al Khaimah, UAE.

\section{Case History}

33-year-old Male Presented to Out Patient Department (OPD) Clinic in Ibrahim Bin Hamad Obaidullah (IBHO) Hospital, Ras Al Khaimah, UAE complaining of a 46 days fever. According to the patient, the fever started 40 days after the ingestion of unpasteurized raw camel milk. The fever was intermittent throughout the day and present every day, accompanied by excessive night sweats, rigors, chills, generalized headache throughout the day, lethargy, myalgia, arthralgia involving multiple joints including ankle and right hip which was of $9 / 10$ in severity in the absence of physical activity, neither swelling nor redness noticed. After 7 days of fever, the patient complained of backache that was gradual on onset radiating to the right buttock, which worsened and made him incapacitated, feeling stiffness in the lower back. The patient experienced neck stiffness, photophobia, and phonophobia. During the course of the disease, the patient developed non-bloody watery diarrhea that lasted for 5 days, and was not accompanied by abdominal cramps. Apart from the above episodes, the patient had previously been well and had a healthy lifestyle, as he was a gym instructor.

\section{Clinical Examination}

The patient was aware and oriented, Glasgow Coma Scale (GCS) 15/15, and hemodynamically stable. On general examination, the patient was found to be irritable, febrile with a fever of $38.7{ }^{\circ} \mathrm{C}$ and heart rate of 110 $\mathrm{bpm}$ at the time of examination. There was no lymphadenopathy or other significant findings. On systemic examination, the patient had stiffness of neck and a positive kerning's sign and deep tendon reflexes were brisk. Other systems were found to be normal.

Serological tests for Brucella melitenisis showed a total antibody titer of 1:5120. Full blood count rates showed high Lymphocytes (68\%), and high Erythrocyte Sedimentation Rate (44.0). Serological tests for Treponema palladium, CMV, EBV, HIV, TB infection were Negative.

The clinical findings and presentation raised the suspicion of CNS infection. Lumbar puncture Investigations showed low Glucose and high Protein in CSF (668.8 mg/dl). 
CSF and blood Culture confirmed Brucella melitenisis bacteria involvement. CT scan showed no abnormalities (Fig. 1). The patient was admitted to the hospital with brucellosis with possible CNS involvement. During hospitalization, patient developed testicular pain, diagnosed as orchitis.

\section{DISCUSSION \\ Clinical features and diagnosis}

The patient has a history of Unpasteurized raw milk ingestion which most likely the mode of infection and the reason behind his symptoms. Neurobrucellosis is a rare complication of human brucellosis, which may present with only CNS symptoms. Nevertheless, the criteria of definitive diagnosis requires one of the following 4 points. is unexplained

Abnormal neurological functioning that High lymphocyte and Protein count in CSF

Positive CSF culture for Brucella or Positive Brucella IgG titer

CSF- response to chemotherapy with a significant drop in the CSF lymphocyte count and protein concentration.

However, not all presentations are similar. In this case, the patient presented with high fever and headache as a chief complaint, and still fulfilled all the criteria to be diagnosed as Neurobrucellosis.

In a study that was done in Turkey, they found that the clinical presentations started with headache in $85 \%$ of the cases ${ }^{7}$. Many patients also do present with symptoms like blurred vision, loss of hearing, behavioral changes, agitations, muscular weakness, disorientation, changes in deep tendon reflexes and paresthesia as reported in some studies ${ }^{7}$. Abducens, facial, and vestibulocochlear cranial nerves were affected more than other cranial nerves in neurobrucellosis ${ }^{7}$.

Another Case Reported in 2016 in India Showed a similar presentation of our case involving an insidious long standing fever for the duration of three months which was associated with progressive weakness of both lower limbs, which fulfilled the criteria and was diagnosed as Neurobrucellosis ${ }^{8}$. The most common neurological findings were PNS followed by meningitis, cranial nerve damage, and meningoencephalitis ${ }^{7}$.

The clinical indicators of human brucellosis remain variable and are not so specific. It may mimic non-infectious or an infectious disease. Clinical range varies from very mild fever to involvement of multisystem. Osteoarticular complication is the main problem associate with morbidity. Death due to brucellosis, though not common, could be attributed to endocarditis and central nervous system involvement ${ }^{9,10}$.

Brucellosis is diagnosed mainly based on clinical indicators and laboratory tests. In laboratory diagnosis, rapid serological tests are preferred over culture methods. In serology, tests such as, indirect fluorescence antibody (IFA) test, standard agglutination tube (SAT) test (primary test) \& enzyme-linked immunosorbent assay (ELISA) are employed. Polymerase chain reaction (PCR) technique due to its specificity and sensitivity is preferred for detection of Brucella $\operatorname{spp}^{9,10}$.

Different manifestations in MRI including periventricular hemorrhage, white matter change, and meningeal enhancement, tentorial enhancement on brain CT scan, basal and dural meningeal enhancement on brain MRI were recorded in the literature as well ${ }^{7-10}$.

In this case, the patient's CT results

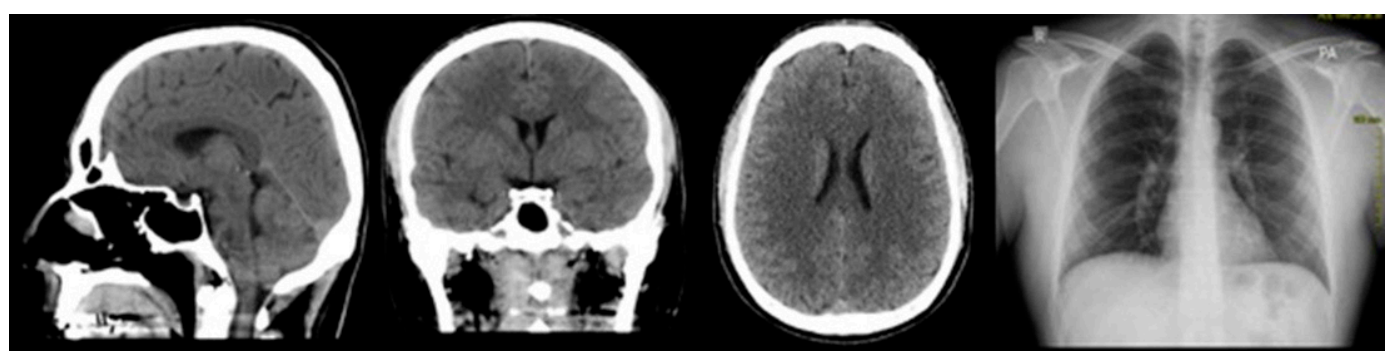

Fig. 1. CT scan and chest $X$-ray of a case with neurobrucellosis related to the consumption of unpasteurized camel milk. CT scan and chest X-ray showed no abnormalities 
were insignificant with no abnormalities and the patient's Chest X-ray was insignificant with no abnormalities (Fig. 1).

\section{Discussion related to treatment aspects}

The goal of therapy in Brucella infection is control of symptoms as early as possible to prevent complications, and monitoring to prevent relapses. There is no unanimity for the choice of antibiotic, duration and dose of the management for neurobrucellosis. Double- or triple-combination treatment with doxycycline, trimethoprimsulfamethoxazole, rifampicin, streptomycin, or ceftriaxone for $>2$ months (3-6 months) has been recommended ${ }^{6}$.

Short-course steroid treatment has been found to be effective in reducing the lasting scarcities in those with optic neuritis, arachnoiditis and multiple-sclerosis-like presentation ${ }^{11}$. Sequela among survivors even with appropriate antibiotic treatment are well known. They are significant if the patient has diffuse CNS, spinal cord or encephalitis association compared to meningitis as a presentation. They have been reported as hearing loss, hemiparesis, aphasia and visual impairment. Though mortality is not very common ${ }^{6,12-14}$.

This patient was treated with ceftriaxone $2 \mathrm{~g}$ twice a day, which is the drug of choice for CNS brucellosis. Alongside with doxycycline $100 \mathrm{mg}$ twice a day and rifampicin 600mg once a day, intravenously for 2 weeks. The patient recovered and was discharged on oral trisulfamethasone, rifampicin 600 and doxycycline 100 twice a day. The mortality rates due to neurobrucellosis has been reported to vary between $0 \%$ - $27 \%$ depending on the severity of the symptoms and the immunocompetency of the patient. However, Sequelae were reported among survivors despite appropriate therapy in some studies ${ }^{15}$. In our Case, the patient was followed up and showed no sequelae or relapse.

\section{CONCLUSION}

In countries like UAE and neighboring countries, brucellosis has been a significant infection as per native surveillance data or innovative e-notification. Leisure connected activities and occupation poses enhanced exposure to brucellosis. UAE has been continuously working on implementing control measures to eradicate Brucella infection among the livestock. There has to be a more complete understanding of the epidemiology of the disease in humans and animals. These facts could contribute to strengthening the public health management of brucellosis. The hospital setups should consider the possibility of infected camel milk ingestion as the mode of infection in brucellosis.

\section{ACKNOWLEDGEMENTS}

None.

\section{CONFLICT OF INTEREST}

The authors declare that there is no conflict of interest.

\section{AUTHORS' CONTRIBUTIONS}

All authors listed have made a substantial, direct and intellectual contribution to the work, and approved it for publication.

\section{FUNDING}

None.

\section{DATA AVAILABILITY}

All datasets generated or analyzed during this study are included in the manuscript.

\section{ETHICS STATEMENT}

This study was approved by Institutional Ethical Committee. A signed informed consent from patient was obtained for this study.

\section{REFERENCES}

1. Al Shehhi N, Aziz F, Al Hosani F, Aden B, Blair I. Human brucellosis in the Emirate of Abu Dhabi, United Arab Emirates, 2010-2015. BMC Infect Dis. 2016; 16(1): 558. https://doi.org/10.1186/s12879-016-1900-9

2. Goncalves DD, Benitez A, Lopes-Mori FM, Alves LA, Freire RL, Navarro IT, Santana MA, Dos Santos LR, Carreira T, Vieira ML, de Freitas JC. Zoonoses in humans from small rural properties in Jataizinho, Parana, Brazil. Braz J Microbiol 2013; 44(1): 125-131. https://doi. org/10.1590/S1517-83822013005000011

3. Maurelio A P V, Santarosa B P, Ferreira D O L, Martins M T A, Paes A C, Megid J. Human brucellosis epidemiological situation in the world. Veterinaria $e$ Zootecnia 2016; 23(4).

4. Bezerra S S, Kim P De C P, Santos FJ De S, Castro K N De C, Lira N S C, Mendes E S. Detection of Brucella spp. in artisan cheese commercialized in Parnaiba, Piaui state, Brazil. Medicina Veterinaria (UFRPE), Recife 2019; 13(1): 33-37. https://doi.org/10.26605/medvetv13n1-2606

5. Pappas G, Akritidis N, Bosilkovski M, Tsianos E. Medical 
progress Brucellosis. N Engl J Med. 2005; 352(22): 2325-67. https://doi.org/10.1056/NEJMra050570

6. Guven T, Ugurlu K, Ergonul O, Celikbas A K, Gok S E, Comoglu S, Baykam N, Dokuzoguz B. Neurobrucellosis: clinical and diagnostic features. Clin Infect Dis. 2013; 56(10): 1407-12. https://doi.org/10.1093/cid/cit072

7. Ceran N, Turkoglu R, Erdem I, Inan A, Engin D, Tireli H, Goktas P. Neurobrucellosis: clinical, diagnostic, therapeutic features and outcome. Unusual clinical presentations in an endemic region. Braz J Infect Dis. 2011; 15(1): 52-9. https://doi.org/10.1016/S14138670(11)70140-4

8. Raina S, Sharma A, Sharma R, Bhardwaj A. Neurobrucellosis: A Case Report from Himachal Pradesh, India, and Review of the Literature. Case Rep Infect Dis. 2016; 2016: https://doi. org/10.1155/2016/2019535

9. Ulu-Kilic A, Metan G, Alp E. Clinical presentations and diagnosis of brucellosis. Recent Pat Antiinfect Drug Discov. 2013 Apr;8(1):34-41. https://doi. org/10.2174/1574891X11308010007
10

Doganay M, Aygen B. Human brucellosis: an overview. Int J Infect Dis 2003; 7: 173-82. https://doi. org/10.1016/S1201-9712(03)90049-X

11. Lulu AR, Araj G F, Khateeb M I, Mustafa M Y, Yusuf A R, Fenech F F. Human brucellosis in Kuwait: a prospective study of 400 cases. Q J Med. 1998; 66(1): 39-54.

12. Kochar D K, Gupta B K, Gupta A, Kalla A, Nayak K C, Purohit S K. Hospital based case series of 175 cases of serologically confirmed Brucellosis in Bikaner. J Assoc Physicians India. 2007; 48(4): 170-3.

13. Shakir R A, Al-Din A S N, Araj G F, Lulu A R, Mousa A R, Saadah M A. Clinical categories of neurobrucellosis: a report on 19 cases. Brain. 1987; 110(1): 213-223. https://doi.org/10.1093/brain/110.1.213

14. Karsen H, Koruk S T, Duygu F, Yapici K, Kati M. Review of 17 cases of neurobrucellosis: clinical manifestations, diagnosis, and management. Arch Iran Med. 2012; 15(8): 491-494.

15. Bodur H, Erbay A, Akinci E, Colpan A, Cevik MA, Balaban N. Neurobrucellosis in an endemic area of brucellosis, Scand J infect Dis. 2003; 35: 94-97. https:// doi.org/10.1080/0036554021000027000 